\title{
A NOTE ON THE TOPOLOGICAL DEGREE AT A CRITICAL POINT OF MOUNTAINPASS-TYPE
}

\author{
HELMUT HOFER
}

\begin{abstract}
In this note we will show that the local degree at an isolated critical point given by the Ambrosetti-Rabinowitz mountainpass-theorem is - 1 for a large class of functionals. This class includes for example those functionals arising in the study of second order elliptic equations.
\end{abstract}

1. The mountainpass-theorem [1] is an elementary but very useful result which has been applied in various situations. Our result establishes a connection between variational and topological methods, which is very useful in studying multiple solutions of partial differential equations. Combining our results with the fact that the local degree at an isolated local minimum is 1 for an admissible functional, see [2] or [3], one obtains a powerful tool. How this tool can be used can be seen in a recent paper of the author [4]. In [4], however, one also needed an order structure and the operators which have been studied were order preserving. This hypothesis is removed here.

Suppose $F$ is a real Banach space and $U$ a nonempty open subset. Assume $\Phi \in C^{1}(U, \mathbf{R})$ and $c, d \in \mathbf{R}$. Define

$$
\begin{gathered}
\operatorname{Cr}(\Phi, c):=\left\{u \in U \mid \Phi^{\prime}(u)=0 \text { and } \Phi(u)=c\right\}, \quad \operatorname{Cr}(\Phi):=\bigcup_{e \in \mathbf{R}} \operatorname{Cr}(\Phi, e), \\
\Phi^{d}:=\Phi^{-1}((-\infty, d]), \quad \Phi_{c}:=\Phi^{-1}([c,+\infty)), \\
\Phi_{c}^{d}:=\Phi^{d} \cap \Phi_{c} \text { and } \dot{\Phi}^{d}:=\Phi^{-1}((-\infty, d)) .
\end{gathered}
$$

Let $X$ be a topological space and $A \subset X$. By int ${ }_{X}(A)$ we denote the interior of $A$ in $X$.

We say that $\Phi \in C^{1}(F, \mathbf{R})$ satisfies the Palais-Smale condition if the following holds:

If $\Phi^{\prime}\left(u_{n}\right) \rightarrow 0$ and $\Phi\left(u_{n}\right) \rightarrow d \in \mathbf{R}$ for some sequence $\left(u_{n}\right) \subset F$, then

$\left(u_{n}\right)$ is precompact.

We need the following

Definition 1. Let $U$ be a nonempty open subset of a Banach space $F$ and $\Phi \in C^{1}(U, \mathbf{R})$. Suppose $u_{0} \in \operatorname{Cr}(\Phi, d)$ for some $d \in \mathbf{R}$. We say that $u_{0}$ is of mountainpass-type (mp-type) if for all open neighbourhoods $W \subset U$ of $u_{0}$ the topological space $W \cap \dot{\Phi}^{d}$ is nonempty and not path-connected.

Received by the editors January 10, 1983.

1980 Mathematics Subject Classification. Primary 58E05; Secondary 47H15, 57H10, 34G20.

Key words and phrases. Critical point theory, Leray-Schauder degree, nonlinear functional analysis.

(C)1984 American Mathematical Society $0002-9939 / 84 \$ 1.00+\$ .25$ per page 
Our first result is a variant of the result in [1]. It shows the existence of a critical point mp-type.

THEOREM 1. Let $\Phi \in C^{1}(F, \mathbf{R})$ satisfy (PS) and assume $e_{0}, e_{1}$ are distinct points in $F$. Define $A, c, d$ by

$$
\begin{aligned}
& A:=\left\{a \in C([0,1], F) \mid a(i)=e_{i}, i=0,1\right\}, \\
& d:=\inf _{a \in A} \sup \Phi(|a|), \text { where }|a|:=a([0,1]), \\
& c:=\max \left\{\Phi\left(e_{0}\right), \Phi\left(e_{1}\right)\right\} .
\end{aligned}
$$

Then, if $d>c$ the set $\operatorname{Cr}(\Phi, d)$ is nonempty. If in addition the critical points in $\operatorname{Cr}(\Phi, d)$ are isolated in $F$ there exists $u_{0}$ of mp-type in $\operatorname{Cr}(\Phi, d)$.

The hypothesis that the critical points in $\operatorname{Cr}(\Phi, d)$ are isolated is necessary as simple examples in $F=\mathbf{R}$ show. In order to study the local degree at an isolated critical point of mp-type we have to impose additional hypotheses on $\Phi$ and $F$.

Let $F$ be a real Hilbert space and $\Phi \in C^{2}(U, \mathbf{R})$, and assume the gradient $\Phi^{\prime}$ of $\Phi$ has the form identity-compact. Furthermore suppose that for all $u_{0} \in \operatorname{Cr}(\Phi)$ the first (smallest) eigenvalue $\lambda_{1}$ of the linearisation $\Phi^{\prime \prime}\left(u_{0}\right) \in L(F)$ at $u_{0}$ is simple provided $\lambda_{1} \leqslant 0$.

We have

THEOREM 2. Let $(\Phi)$ hold and assume $u_{0} \in U$ is an isolated critical point of mp-type. Then the local degree at $u_{0}$ is -1 .

REMARK. Moreover, one can compute the homology $H$ of the pair $\left(\Phi^{d}, \Phi^{d} \backslash\left\{u_{0}\right\}\right)$, $d=\Phi\left(u_{0}\right)$. It turns out that $H=(\mathbf{Z}, 1)$. Again Theorem 2 follows from this by a generalisation of a result of Rothe [5]. Namely, for all $C^{2}$-functionals having a gradient of the form identity-compact we have the following relation between the local degree "deg" at $u_{0}$ and the homology $H$ of the above pair: deg $=\chi(H)$, where $\chi$ denotes the Euler-characteristic. This relation is true without the hypothesis $(\mathrm{H})$ imposed by Rothe.

Before proving the results we give an example of a functional satisfying $(\Phi)$. Consider the partial differential equation

$$
-\Delta u=f(\cdot, u) \quad \text { in } \Omega, \quad u=0 \quad \text { on } \partial \Omega,
$$

where $\Omega \subset \mathbf{R}^{n}, n \geqslant 3$, is a bounded domain having a smooth boundary $\partial \Omega$. Assume $f \in C^{1}(\bar{\Omega} \times \mathbf{R}, \mathbf{R})$ and $f^{\prime}(x, s):=\partial_{s} f(x, s)$ satisfies the growth-condition

$$
\left|f^{\prime}(x, s)\right| \leqslant c\left(1+|s|^{\sigma-1}\right) \quad \forall(x, s) \in \bar{\Omega} \times \mathbf{R},
$$

where $c>0$ and $\sigma \in\left[1,(n+2)(n-2)^{-1}\right)$. Using $L^{p}$-estimates and the Sobolevembedding-theorems it follows that the solutions of (1) are exactly the critical points of the $C^{2}$-functional $\Phi \in C^{2}(F, R), F:=H_{0}^{1}(\Omega)$, defined by

$$
\Phi(u)=2^{-1}\|u\|^{2}-\int_{\Omega} \hat{f}(\cdot, u)
$$


where $(u, v)=\int_{\Omega}\langle\nabla u, \nabla v\rangle,\|u\|=(u, u)^{1 / 2}$, and $\hat{f}(x, s):=\int_{0}^{s} f(x, t) d t$. Let $u_{0}$ be a critical point of $\Phi$. Hence $u_{0} \in C^{2}(\bar{\Omega})$. Denote by $\lambda_{1}$ the first eigenvalue of $\Phi^{\prime \prime}\left(u_{0}\right)$ and suppose $\lambda_{1} \leqslant 0$. Define $\mu:=\left(1-\lambda_{1}\right)^{-1} \in(0,1]$ and $b \in C(\bar{\Omega})$ by $b(x):=f^{\prime}\left(x, u_{0}(x)\right)$. We find an element $v \neq 0$ in $F$ such that

$$
-\Delta v=\mu b v \text { in } \Omega, \quad v=0 \text { on } \partial \Omega \text {. }
$$

Hence, $\mu \int_{\Omega} b v^{2}>0$, and since $\mu>0$ we infer that $b\left(x_{0}\right)>0$ for some $x_{0} \in \Omega$. By results of Manes-Micheletti [6], or Hess-Kato [7], the eigenvalue problem

$$
-\Delta u=\hat{\lambda} b u \quad \text { in } \Omega, \quad u=0 \quad \text { on } \partial \Omega
$$

possesses a smallest positive eigenvalue $\hat{\lambda}_{1}$ with a one-dimensional eigenspace spanned by some $u_{1}$ not changing sign. Now our assertion follows from the fact that $\hat{\lambda}_{1}=\mu$.

2. Proof of the theorems. In order to prove Theorem 1 the following class $D(\Phi)$ of homotopies of the identity is important in the study of the critical points of $\Phi$.

Definition 2. Denote by $D(\Phi)$ the set consisting of all continuous maps $\sigma$ : $[0,1] \times F \rightarrow F$ such that $\sigma(0, \cdot)=$ Id and $t \rightarrow \Phi(\sigma(t, u))$ is nonincreasing for all $u \in F$.

The following lemma is a trivial variant of the standard deformation-lemma [8]:

Lemma 1. Given $\bar{\varepsilon}>0, d \in \mathbf{R}$ and open neighbourhoods $W$ and $V$ of $\operatorname{Cr}(\Phi, d)$, where $\Phi \in C^{1}(F, \mathbf{R})$, such that $W \supset \bar{V}$ and $\operatorname{dist}(\partial W, V)>0$, there exist $\varepsilon \in(0, \bar{\varepsilon}]$ and $\sigma \in D(\Phi)$ with

$$
\begin{gathered}
\sigma\left(\{1\} \times\left(\Phi^{d+\varepsilon} \backslash V\right)\right) \subset \Phi^{d-\varepsilon}, \\
\sigma([0,1] \times \bar{V}) \subset W, \\
\sigma(t, u)=u \quad \forall(t, u) \in[0,1] \times\left(\Phi_{d+\bar{\varepsilon}} \cup \Phi^{d-\bar{\varepsilon}}\right) .
\end{gathered}
$$

Sketch of The Proof. The assertions (2) and (4) are well known. In order to see (3) observe that $\sigma$ is the restriction of a flow associated to an ordinary differential equation of the form $\dot{\sigma}=G \sigma$ in $F$ with $\|G u\| \leqslant 1 \forall u \in F$. Hence $\|\sigma(t, u)-u\| \leqslant|t|$ $\forall(t, u) \in \mathbf{R} \times F$. This implies for some $\delta>0$ that $\sigma([0, \delta] \times \bar{V}) \subset W$. Then making a reparametrisation of the time $t$ one obtains the desired map $\sigma$. If necessary one has of course to replace $\varepsilon$ by some smaller positive constant.

Proof of THE THEOREM 1. Arguing indirectly we may assume by (PS) that $\operatorname{Cr}(\Phi, d)$ contains only a finite number of critical points all being not of mp-type. Further we may assume arguing as in [1], that $\operatorname{Cr}(\Phi, d) \neq \varnothing$. By our hypothesis all the critical points in $\operatorname{Cr}(\Phi, d)$ are isolated in $F$. Let $\operatorname{Cr}(\Phi, d)=\left\{u_{1}, u_{2}, \ldots, u_{n}\right\}$. We find corresponding open neighbourhoods $U_{i}, u_{i} \in U_{i}$, such that $U_{i} \cap \dot{\Phi}^{d}$ is either empty or path-connected and $U:=\cup U_{i} \supset \operatorname{Cr}(\Phi, d)$. Define $\delta>0, \bar{\varepsilon}>0, W$ and $V$ by

$$
\begin{aligned}
& \bar{\varepsilon}:=2^{-1}(d-c), \\
& \delta:=8^{-1} \min \{\operatorname{dist}((\partial U) \cup\left.\left\{e_{0}, e_{1}\right\}, \operatorname{Cr}(\Phi, d)\right), \\
&\left.\inf \left\{\operatorname{dist}\left(u_{i}, \operatorname{Cr}(\Phi) \backslash\left\{u_{i}\right\}\right) \mid i=1, \ldots, n\right\}\right\},
\end{aligned}
$$




$$
\begin{aligned}
& W:=\{u \in F \mid \operatorname{dist}(u, \operatorname{Cr}(\Phi, d))<2 \delta\}, \\
& V:=\{u \in F \mid \operatorname{dist}(u, \operatorname{Cr}(\Phi, d))<\delta\} .
\end{aligned}
$$

Given $\bar{\varepsilon}>0, W$ and $V$ as above we find by Lemma $1, \varepsilon \in(0, \bar{\varepsilon}]$ and $\sigma \in D(\Phi)$ satisfying (2)-(4). Choose $a \in A$ with $|a| \subset \Phi^{d+\varepsilon}$. Note that $W=\cup W_{i}$ and $V=$ $\cup V_{i}$, where $W_{i}$ and $V_{i}, i=1, \ldots, n$, are open $(2 \delta)$ - or $\delta$-balls, respectively, around $u_{i}$. Define $M:=\{t \in[0,1] \mid a(t) \notin V\}$ and $\Gamma$ by

$$
\Gamma:=\left(U \cap \dot{\Phi}^{d}\right) \cup \sigma(\{1\} \times a(M)) .
$$

Observe that $e_{0}, e_{1} \in \Gamma$. Denote by $\tilde{\Gamma}$ the path-component of $\Gamma$ containing $e_{0}$. We shall show that $e_{1} \in \tilde{\Gamma}$. Since $\tilde{\Gamma} \subset \Gamma \subset \dot{\Phi}^{d}$ this contradicts the definition of $d$. Obviously we may assume that $M \neq[0,1]$ because otherwise we are done. Define

$$
t_{0}:=\sup \{t \in M \mid \sigma(1, a(t)) \in \tilde{\Gamma}\} .
$$

We have to show that $t_{0}=1$. Suppose $t_{0}<1$. Since $0 \in \operatorname{int}_{[0,1]} M$ this implies $t_{0} \in(0,1)$. Denote by $\left[t^{-}, t^{+}\right]$the component in $M$ containing $t_{0}$. We must have $t_{0}=t^{+}$because in the case $t_{0}<t^{+}$we immediately obtain a contradiction to the definition of $t_{0}$. Therefore $a\left(t_{0}\right) \in \partial V$. There exists a unique $i_{0} \in\{1, \ldots, n\}$ such that $\left\|a\left(t_{0}\right)-u_{i_{0}}\right\|=\delta$. Define

$$
\hat{t}:=\sup \left\{t \in\left[t_{0}, 1\right] \mid a(t) \in \bar{V}_{i_{0}}\right\} .
$$

By the preceding discussion, since $t_{0}=t^{+}$we find $\hat{t} \in\left(t_{0}, 1\right)$. Of course $a(\hat{t}) \in \partial V_{i_{0}}$. Hence $\hat{t} \in M$ and moreover $g_{1}:=\sigma(1, a(\hat{t})) \in W_{i_{0}} \cap \dot{\Phi}^{d} \subset U_{i_{0}} \cap \dot{\Phi}^{d}$. Moreover $g_{2}:=\sigma\left(1, a\left(t_{0}\right)\right) \in U_{i_{0}} \cap \dot{\Phi}^{d}$. Since $U_{i_{0}} \cap \tilde{\Phi} \dot{\Phi}^{d}$ is path-connected $g_{1}$ and $g_{2}$ are in the same path-component. Using the $g_{2} \in \tilde{\Gamma}$ we conclude that $g_{1} \in \tilde{\Gamma}$. Therefore we obtain the contradiction $t_{0} \geqslant \hat{t}>t_{0}$.

Now we come to the proof of Theorem 2. We need the following variant of the Morse-lemma. The proof uses a combination of ideas due to Gromoll and Meyer [9], Takens [10], and the author [4, Lemma 5].

Lemma 2. Let $F$ be a real Hilbert space and $\Phi \in C^{2}(U, \mathbf{R})$ having a gradient of the form identity-compact. Suppose 0 is an isolated critical point of $\Phi$ with $\Phi(0)=0$. Let $F=F^{-} \oplus F^{0} \oplus F^{+}$be the canonical decomposition associated to $\Phi^{\prime \prime}(0)$ via the spectral resolution.

Then there exist an origin-homeomorphism $D$ defined on a 0-neighbourhood into $F$ and an origin-preserving $C^{1}$-map $\beta$ defined on a 0 -neighbourhood in $F^{0}$ into $F^{-} \oplus F^{+}$ such that

$$
\begin{gathered}
\Phi(D u)=-2^{-1}\|x\|^{2}+2^{-1}\|z\|^{2}+\Phi(\beta y+y), \\
\left(P^{-}+P^{+}\right) \Phi^{\prime}(\beta y+y)=0,
\end{gathered}
$$

for all $u=x+y+z,\|u\|$ small. Moreover we have for the local degrees the formula

$$
\operatorname{deg}_{\text {loc }}\left(\Phi^{\prime}, 0\right)=(-1)^{\operatorname{dim}\left(F^{-}\right)} \operatorname{deg}_{\text {loc }}\left(\Psi^{\prime}, 0\right)
$$

where $\Psi(y):=\Phi(\beta y+y)$. 
Sketch of the proof. By the implicit function theorem we find a constant $\delta>0$ and a map $\beta$ : $F^{0} \cap B_{\delta} \rightarrow F^{-} \oplus F^{+}$such that (6) holds. We define a map $\tilde{\Phi}$ : $[0,1] \times\left(F^{-} \oplus\left(B_{\delta} \cap F^{0}\right) \oplus F^{+}\right) \rightarrow \mathbf{R}$ by

$$
\tilde{\Phi}(t, u)=t \Phi(u+\beta y)+(1-t)\left(\Phi(y+\beta y)-2^{-1}\|x\|^{2}+2^{-1}\|z\|^{2}\right) .
$$

One can derive easily the following estimates, denoting by $\tilde{\Phi}_{t}$ and $\tilde{\Phi}_{u}$ the gradients with respect to the variables $t$ and $u$, respectively:

$$
\begin{gathered}
\left|\tilde{\Phi}_{t}(t, u)\right| \leqslant c_{1}\|v\|^{2}, \quad\|u\|<\tilde{\delta} \quad\left(c_{1}>0\right), \\
c_{2}\|v\| \leqslant\left\|\tilde{\Phi}_{u}(t, u)\right\| \leqslant c_{3}\|u\|, \quad\|u\|<\tilde{\delta} \quad\left(c_{2}, c_{3}>0\right)
\end{gathered}
$$

where $\tilde{\delta} \in(0, \delta]$ is a suitable constant and $v=\left(P^{-}+P^{+}\right) u$. Now we introduce a vectorfield $G:[0,1] \times B_{\tilde{\delta}} \rightarrow H$ by

$$
G(t, u):=\left[\begin{array}{l}
0, \quad \text { for all } u \text { with } \tilde{\Phi}_{u}(t, u)=0, \\
-\tilde{\Phi}_{t}(t, u)\left\|\tilde{\Phi}_{u}(t, u)\right\|^{-2} \tilde{\Phi}_{u}(t, u), \quad \text { otherwise. }
\end{array}\right.
$$

A straightforward but tedious computation using the previous estimates shows that $G$ is continuous and that there exists a constant $M>0$ such that

$$
\left\|G(t, u)-G\left(t, u^{\prime}\right)\right\| \leqslant M\left\|u-u^{\prime}\right\| \quad \text { for all } u, u^{\prime} \in B_{\tilde{\delta}}, t \in[0,1] .
$$

By the standard existence and uniqueness results for ordinary differential equations we find $\sigma, 0<\sigma \leqslant \tilde{\delta}$, such that for all initial values $u \in B_{\sigma}$ the solution of the ODE,

$$
\dot{\eta}_{u}=G\left(t, \eta_{u}\right), \quad \eta_{u}(0)=u
$$

exists for $t \in[0,1]$. Define $D: B_{\sigma} \rightarrow H$ by $D u:=\eta_{u}(1)$. Obviously $D$ is a homeomorphism of $B_{\sigma}$ onto an open neighbourhood of 0 . Further we calculate $d \tilde{\Phi}\left(t, \eta_{u}(t)\right) / d t$ $=0$. Hence we find with $u=x+y+z$,

$$
\begin{aligned}
\Phi(D u) & =\tilde{\Phi}\left(1, \eta_{u}(1)\right)=\tilde{\Phi}\left(0, \eta_{u}(0)\right) \\
& =\Phi(y+\beta y)-2^{-1}\|x\|^{2}+2^{-1}\|z\|^{2} .
\end{aligned}
$$

This completes the proof of (5) and (6). In order to prove (7) we define a homotopy $h^{*}:[0,1] \times \bar{B}_{\sigma} \rightarrow H$ by

$$
h^{*}(t, u):=\left[\begin{array}{lr}
\left(P^{-}+P^{+}\right) \Phi^{\prime}(u)+P^{0} \Phi^{\prime}(t \beta y+(1-t)(x+z)+y), & t \in[0,1], \\
\left(P^{-}+P^{+}\right) \Phi^{\prime}(x+z+(2-t) y)+P^{0} \Phi^{\prime}(y+\beta y), \quad t \in[1,2], & t(3-t)\left(P^{-}+P^{+}\right) \Phi^{\prime}(x+z)+(t-2)(-x+z)+P^{0} \Phi^{\prime}(y+\beta y), \\
& t \in[2,3] .
\end{array}\right.
$$

Since $\operatorname{dim}\left(F^{-}\right)+\operatorname{dim}\left(F^{0}\right)<\infty$ one easily sees that $h^{*}$ is a homotopy of the form identity-compact. Moreover there exists $\varepsilon_{0}>0$ such that there exists no solution of $h(t, u)=0$ with $\|u\|=\varepsilon, t \in[0,1]$, for all $\varepsilon \in\left(0, \varepsilon_{0}\right]$. This implies by the properties of the degree the statement (7). 
Proof of Theorem 2. We may assume $u_{0}=0, \Phi(0)=0$, and by Lemma 2 that $\Phi$ has the form

$$
\Phi(u)=-2^{-1}\|x\|^{2}+2^{-1}\|z\|+\Psi(y), \quad u=x+y+z,
$$

where 0 is an isolated critical point of $\Psi$. Further let $W=W^{-} \oplus W^{0} \oplus W^{+}$, $\bar{W} \subset U$, due to our orthogonal decomposition of $F$ such that $W^{-}$and $W^{+}$are $\delta$-balls around 0 and $W^{0}$ is a ball with

$$
|\Psi(y)| \leqslant \delta^{2} / 8 \text { for all } y \in W^{0} .
$$

Moreover we may assume $\operatorname{Cr}(\Phi) \cap \bar{W}=\{0\}$. First of all we show that $\operatorname{dim}\left(F^{-}\right) \leqslant 1$. Arguing indirectly suppose that $\operatorname{dim}\left(F^{-}\right) \geqslant 2$. We will show that $W \cap \dot{\Phi}^{0}=: \Gamma$ is path-connected in that case contradiction our assumption that 0 is of mp-type. We shall write for $g, g^{\prime} \in \Gamma, g \sim g^{\prime}$ iff they are in the same path-component. Let $g=x_{1}+y_{1}+z_{1} \in \Gamma$. We find by (5) $g \sim g_{1}:=x_{1}+y_{1}$.

Now we can choose $x_{2} \in W^{-}$with $\left\|x_{2}\right\|>\delta / 2$ and $\left\|t x_{2}+(1-t) x_{1}\right\| \geqslant\left\|x_{1}\right\|$ $\forall t \in[0,1]$. Hence by (5) $g_{1} \sim g_{2}:=x_{2}+y_{1}$. Now making use of (8) $g_{2} \sim g_{3}:=x_{2}$. Hence we have shown

(9) For every $g \in \Gamma$ there exists $\hat{g} \in \tilde{\Gamma}:=\bar{W} \backslash\{0\}$ with $g \sim \hat{g}$ provided $F^{-} \neq\{0\}$.

If $\operatorname{dim}\left(F^{-}\right) \geqslant 2$ the set $\tilde{\Gamma}$ is path-connected. This implies by the preceding discussion that $\Gamma$ is path-connected which contradicts the fact that 0 is of mp-type. Therefore $\operatorname{dim}\left(F^{-}\right) \leqslant 1$.

Next we will show that the negative Morse-index and the zero Morse-index satisfy

$$
\left(m^{-}, m^{0}\right) \in(\{1\} \times \mathbf{N}) \cup\{(0,1)\}
$$

where $\mathbf{N}:=\{0,1, \ldots\}$. Suppose $\left(m^{-}, m^{0}\right) \notin\{1\} \times \mathbf{N}$. By the preceding discussion $m^{-}=0$. If $m^{0}=0,0$ is a local minimum which cannot be of mp-type. Hence $m^{0} \geqslant 1$. By our assumption $(\Phi)$ the first eigenvalue $\lambda_{1}=0$ is simple. This yields $m^{0}=1$. Therefore $\left(m^{-}, m^{0}\right)=(0,1)$.

Now we come to the heart of the proof. It is divided into two cases. We begin with

$$
\text { If }\left(m^{-}, m^{0}\right) \in\{1\} \times \mathbf{N} \Rightarrow \operatorname{deg}_{\mathrm{loc}}\left(\Phi^{\prime}, 0\right)=-1 .
$$

We have by the properties of the degree

$$
\operatorname{deg}_{\operatorname{loc}}\left(\Phi^{\prime}, 0\right)=-\operatorname{deg}_{\text {loc }}\left(\Psi^{\prime}, 0\right) \text {. }
$$

We will show that 0 is a local minimum of $\Psi$. This implies by results in [2] or [3] that $\operatorname{deg}_{\operatorname{loc}}\left(\Psi^{\prime}, 0\right)=1$. Combining this with (12) will prove (13). Arguing indirectly let us assume that 0 is not a local minimum of $\Psi$. As in the proof of (9) we find that $\Gamma:=W \cap \dot{\Phi}^{0}$ has at most two path-components which can be represented by two elements $\hat{x}, \bar{x} \in W^{-} \backslash B_{(2 \delta / 3)}$. It is enough to show that $\hat{x} \sim \bar{x}$ giving a contradiction. By our assumption we find $y \in W^{0} \backslash\{0\}$ such that $\Psi(y)<0$. We define a path $a$ : $[0,3] \rightarrow \Gamma$ joining $\hat{x}$ and $\bar{x}$ by

$$
a(t):=\left[\begin{array}{ll}
\hat{x}+t y, & t \in[0,1] \\
(2-t) \hat{x}+(t-1) \bar{x}+y, & t \in[1,2], \\
\bar{x}+(3-t) y, & t \in[2,3]
\end{array}\right.
$$


The remaining case is $\operatorname{dim}\left(F^{-}\right)=0$ or equivalently $\left(m^{-}, m^{0}\right)=(0,1)$ by $(10)$. We show

$$
\text { If }\left(m^{-}, m^{0}\right)=(0,1) \Rightarrow \operatorname{deg}_{\mathrm{loc}}\left(\Phi^{\prime}, 0\right)=-1 .
$$

It is enough to prove that 0 is a local maximum of $\Psi$. Since $\operatorname{dim}\left(F^{0}\right)=1$ this will imply that $\operatorname{deg}_{\mathrm{loc}}\left(\Psi^{\prime}, 0\right)=-1$. Using that 0 is an isolated critical point of $\Psi$ there are only four types of behaviour of $\Psi$ namely $\Psi \sim y^{2}, \Psi \sim-y^{2}, \Psi \sim \operatorname{sign}(y) y^{2}$, and $\Psi \sim-\operatorname{sign}(y) y^{2}$. If $\Psi \sim y^{2}, 0$ is a local minimum of $\Phi$ which is impossible. If $\Psi$ behaves like $\operatorname{sign}(y) y^{2}$ it easily follows that $\Gamma$ is contractible to a point. In particular $\Gamma$ is path-connected. A similar argument holds if $\Psi \sim-\operatorname{sign}(y) y^{2}$. Hence the only possible case is $\Psi \sim-y^{2}$. But this is exactly the case when 0 is a local maximum and we are done.

\section{REFERENCES}

1. A. Ambrosetti and P. Rabinowitz, Dual variational methods in critical point theory, J. Funct. Anal. 14 (1973), 343-381.

2. H. Amann, A note on the degree theory for gradient mappings, Proc. Amer. Math. Soc. 84 (1982), 591-595.

3. P. H. Rabinowitz, A note on the topological degree for potential operators, J. Math. Anal. Appl. 51 (1975), 483-492.

4. H. Hofer, Variational and topological methods in partially ordered Hilbert spaces, Math. Ann. 261 (1982), 493-514.

5. E. H. Rothe, $A$ relation between the type numbers of a critical point and the index of the corresponding field of gradient vectors, Math. Nachr. 4 (1951), 12-27.

6. A. Manes and A. M. Micheletti, Un'extensione della teoria variazionale classica degli autovalori per operatori ellittici del secondo ordine, Boll. Un. Mat. Ital. 7 (1973), 285-301.

7. P. Hess and T. Kato, On some linear and nonlinear eigenvalue problems with indefinite weight function, Comm. Partial Differential Equations 5 (1980), 999-1030.

8. P. H. Rabinowitz, Variational methods for nonlinear eigenvalue problems, in eigenvalues of nonlinear problems (G. Prodi, Ed.), C.I.M.E. Edizione Cremonese, Rome, 1975, pp. 141-195.

9. D. Gromoll and W. Meyer, On differentiable functions with isolated critical points, Topology 8 (1969), 361-369.

10. F. Takens, A note on sufficiency of jets, Invent. Math. 13 (1971), 225-231.

School of Mathematics, University of Bath, Claverton Down, Bath BA2 7AY, Great Britain 